\title{
Contrast-enhanced ultrasonographic findings in three dogs with lung lobe torsion
}

\author{
Domenico CAIVANO ${ }^{1)}$, Francesco BIRETTONI ${ }^{1)}$, Antonello BUFALARI ${ }^{1)}$, Valentina DE MONTE ${ }^{1)}$, \\ Giovanni ANGELI ${ }^{1)}$, Maria Elena GIORGI ${ }^{1)}$, Valentina PATATA ${ }^{1)}$ and Francesco PORCIELLO ${ }^{1)}$ \\ 1) Department of Veterinary Medicine, University of Perugia, Via San Costanzo, 4-06126 Perugia, Italy
}

(Received 9 July 2015/Accepted 9 October 2015/Published online in J-STAGE 24 October 2015)

ABSTRACT. Lung lobe torsion is rare but life-threatening condition in the dog. Thoracic radiographs and conventional ultrasonography cannot be conclusive for the diagnosis, and computed tomography is useful but is limited by cost and availability. This report describes the findings of contrast-enhanced ultrasonography in $3 \mathrm{dogs}$ with lung lobe torsion. Contrast-enhanced ultrasonography showed the absence or reduction of pulmonary vascularization secondary to twisting of the lung lobe around its bronchovascular pedicle in all three dogs. Moreover, contrast-enhanced ultrasonography distinguished partial pulmonary atelectasis from a lung lobe torsion. These preliminary results suggest that contrast-enhanced ultrasonography can improve the accuracy of conventional ultrasonography for detection of pulmonary blood flow compromise in dogs with lung lobe torsion.

KEY WORDS: canine, CEUS, contrast-enhanced ultrasonography, lung lobe torsion

doi: 10.1292/jvms.15-0417; J. Vet. Med. Sci. 78(3): 427-430, 2016

Lung lobe torsion (LLT) is a pulmonary condition characterized by twisting of a lobe around its bronchovascular pedicle $[4,5,12]$. This causes bronchial obstruction and pulmonary blood flow compromise, resulting in congestion of the lung lobe $[5,12]$. Progressive lobar congestion leads to parenchymal and alveolar edema, hemorrhage and necrosis [5]. While LLT is considered rare in dogs, it is life-threatening and requires surgical lung lobectomy [5, 9, 11, 12, 15]. Therefore, this condition must be accurately distinguished from others with similar signs that do not require surgery. Many diseases can mimic a LLT, including pneumonia, thromboembolism, contusion, atelectasis, neoplasia and coagulopathy [5].

Imaging is critical to make a diagnosis, because clinical signs, hematological results and cytological findings from pleural effusion are nonspecific [12]. Radiographic and ultrasonographic findings of the LLT have been well described in dogs, but these diagnostic tools can be nonspecific $[1,5,10]$. Twisting of a lobe around its bronchovascular pedicle causes pulmonary blood flow compromise, but this change cannot be easily estimated. The study of lung vascularity in LLT using color or power Doppler ultrasonographic modality could be hampered by respiratory motion artifact [5]. Computed tomography (CT) is a useful imaging tool in suspected LLT cases $[15,16]$, but it is not widely available, can be cost prohibitive and requires general anesthesia or deep sedation. Contrast-enhanced ultrasonography (CEUS) is an imaging modality that improves the diagnostic accuracy of conventional ultrasonography (CUS) by increasing the intensity

*Correspondence to: Caivano, D., Department of Veterinary Medicine, University of Perugia, Via San Costanzo, 4-06126 Perugia, Italy. e-mail: domenico.caivano@unipg.it

C2016 The Japanese Society of Veterinary Science

This is an open-access article distributed under the terms of the Creative Commons Attribution Non-Commercial No Derivatives (by-nc-nd) License $<$ http://creativecommons.org/licenses/by-nc-nd/4.0/>. of blood-pool echo signals in arteries and veins through intravenous injection of stabilized gas-filled microbubbles as a contrast agent [8]. In human medicine, CEUS is able to characterize lung vascularity in peripheral lung consolidations $[7,13,14]$. In human and veterinary medicine, CEUS has been used to study the vascularity within different organs $[6,8,17]$, but to the author's knowledge, the use of the CEUS has not been reported for the twisted lung lobe examination.

The purpose of this report was to describe CEUS findings in three dogs with confirmed LLT. We hypothesized that qualitative analysis of the CEUS would detect changes in the vascularization of LLT and provide additional information in the diagnosis of twisted lung lobe.

Three dogs (3-year-old, female Bernese mountain dog; 12-year-old, female Pinscher toy; and 3-year-old male mixed breed dog) were referred to the Veterinary Teaching Hospital of Perugia University for suspected LLT. Clinical, hematological and radiographic findings of the three dogs included in this report are summarized in Table 1. All 3 dogs displayed similar clinical symptoms: dyspnea, anorexia/dysorexia and pleural effusion were present from 1 week (Case 3), 10 days (Case 1) and 3 weeks (Case 2). Chylothorax (Cases 1 and 3) and pure transudate (Case 2) were previously diagnosed by the referring veterinarian. Hematological results showed leukocytosis (in all cases) and hypoproteinemia (Case 2). Thoracic radiographs were suggestive with LLT in case 1 and not conclusive in case 2. Radiographic examination was not performed in case 3 .

For all dogs, both CUS and CEUS were performed with the same ultrasound machine (MyLab Class C, Esaote, Genova, Italy) using 5-8 MHz microconvex (for CUS) and 3-8 MHz linear (for CEUS) transducers. All dogs were placed in lateral or sternal recumbency with only manual restraint. The sonography system was also equipped with a contrast-tuned imaging technology module (CnTI ${ }^{\mathrm{TM}}$, Esaote). All medical procedures were done with the informed consent of the owners and in accordance with Italian laws on animal care. 
Table 1. Signalment, clinical signs, hematologic results and radiographic findings

\begin{tabular}{cllll}
\hline Case & \multicolumn{1}{c}{ Signalment } & \multicolumn{1}{c}{ Clinical signs } & Hematological results & \multicolumn{1}{c}{$\begin{array}{c}\text { Radiographic findings } \\
\text { (TR } \text { by referring veterinarian) }^{\text {a }} \text { by }\end{array}$} \\
\hline 1 & $\begin{array}{l}\text { 3-year-old, female, } \\
\text { Bernese mountain dog }\end{array}$ & $\begin{array}{l}\text { Dyspnea, anorexia and progressive } \\
\text { lethargy, chylothorax (10 days) }\end{array}$ & $\begin{array}{l}\text { Leukocytosis }\left(22 \times 10^{9} / l ;\right. \\
\text { reference interval: } 6 \text { to } 16)\end{array}$ & $\begin{array}{l}\text { Moderate pleural effusion; area of homo- } \\
\text { geneous radiopacity with few signs of air } \\
\text { entrapment in the left apical lung lobe }\end{array}$ \\
\hline 2 & $\begin{array}{l}12 \text {-year-old, female, } \\
\text { Pinscher toy }\end{array}$ & $\begin{array}{l}\text { Dyspnea, anorexia, pleural effusion, Leukocytosis }\left(20 \times 10^{9} / l ;\right. \\
\text { hypoproteinemia secondary to renal } \\
\text { damage (3 weeks) }\end{array}$ & $\begin{array}{l}\text { Moference interval: } 6 \text { to } 16), \\
\text { hypoproteinemia }(3.2 \mathrm{~g} / \mathrm{d} l ; \\
\text { reference interval: } 6 \text { to } 8.5)\end{array}$ & pleural effusion \\
\hline 3 & $\begin{array}{l}\text { 3-year-old, male, mixed } \\
\text { breed }\end{array}$ & $\begin{array}{l}\text { Dyspnea, disorexia, chylothorax } \\
\text { (1 week) }\end{array}$ & $\begin{array}{l}\text { Leukocytosis }\left(23 \times 10^{9} / l ;\right. \\
\text { reference interval: } 6 \text { to } 16)\end{array}$ & NA $^{\text {b) }}$ \\
\hline
\end{tabular}

a) TR: thoracic radiographs, b) NA: not available.

CUS examination showed moderate (Cases 1 and 2) to severe (Case 3) pleural effusion. Suspected twisted lung lobe was hypoechoic, enlarged and hepatized in all dogs. A fluid bronchogram and a presumptive dilated vascular structure appeared in the central portion of the suspected lung lobe in case 1 and case 2 (Figs. 1 and 2). Fluid bronchogram appeared as a linear structure, more echogenic than presumptive dilated vascular structure (Figs. 1 and 2). Air bronchogram within the suspected lung lobe was observed in case 3 (Fig. 3). A second partially consolidated lung lobe with reverberating foci, consistent with gas, was visualized craniomedially to the first lesion in case 1 (Fig. 1). Color Doppler evaluation was non-diagnostic due to respiratory motion artifact in all dogs. Suspected twisted lung lobe was located in the left cranial thorax in case 1, and in the right middle thorax in case 2 and case 3 .

For CEUS, the suspected twisted lobe was centered on the screen, and a single focal zone was placed at the deepest part of the lesion. The mechanical index was set at 0.04-0.05, and the gain was adjusted so that few signals from the surrounding tissues were present. A bolus $(0.03-0.06 \mathrm{ml} / \mathrm{kg})$ of sulfur hexafluoride microbubbles stabilized by a phospholipid shell (SonoVue ${ }^{\circledR}$, Bracco, Italy) was intravenously infused by hand as previously described [2]. Suspected twisted lung lobes were assessed for the presence or absence of enhancement after the injection of the contrast agent. The enhancement patterns were evaluated for $2 \mathrm{~min}$ and compared with the surrounding tissues (other lung lobe, liver or intrathoracic vessels). In case 1, the suspected twisted lung lobe displayed inhomogeneous and mild vascular enhancement after $12 \mathrm{sec}$ from injection of the contrast agent; enhancement was more evident after $28 \mathrm{sec}$ (Fig. 1). The second partially consolidated lung lobe showed homogeneous and marked vascular enhancement (Fig. 1). In case 2, the suspected twisted lung lobe showed absence of vascular enhancement after injection of the contrast agent (Fig. 2). Marked vascular enhancement was visualized in a large vessel (caudal vena cava) at the periphery of the lung lobe after $8 \mathrm{sec}$ from injection of the contrast agent (Fig. 2). In case 3, the suspected twisted lung lobe showed some enhanced vessels inside after $25 \mathrm{sec}$ from injection of the contrast agent (Fig. 3). Marked vascular enhancement was visualized within the liver after $8 \mathrm{sec}$ from injection of the contrast agent (Fig. 3).

Thoracic CT (Siemens, Milano, Italy) was performed prior to surgery to further characterize the lesions in case 2 and case 3. CT confirmed the LLT showing an extended cystic-like lesion characterized by ring-enhancement that involved the right middle lung lobe after contrast medium administration (Case 2) and a consolidated area in the ventral portion of the right medial lung lobe with mild contrastenhancement of the lesion after iodine intravenous injection (Case 3).

The final diagnosis of LLT was made by surgical exploration in all dogs: in case 1 , the left cranial lung lobe was rotated approximately $180^{\circ}$ and appeared to be congested, consolidated and necrotic. Partial atelectasis of adjacent caudal lobe disappeared after the re-inflation using positivepressure ventilation. Left cranial lung lobectomy and thoracic duct ligation were performed; In case 2 , the right middle lung lobe was rotated approximately $360^{\circ}$ and appeared to be congested, consolidated and necrotic. Right middle lung lobectomy was performed; In case 3 , the right middle lung lobe was rotated approximately $360^{\circ}$ and appeared to be congested, consolidated and necrotic. Right middle lung lobectomy and thoracic duct ligation were performed. Histological evaluation of the resected lung lobes was consistent with lung lobe torsion.

To our knowledge, this is the first report describing the use of CEUS in a small group of dogs with LLT. Thoracic radiographs are the imaging technique commonly used to make a suspected diagnosis of LLT $[1,5,10]$. In our dogs, radiographic features were suggestive of LLT in $\operatorname{dog} 1$ and not conclusive in dog 2. Because radiographic findings are not specific, ultrasonography can be a useful imaging modality in the diagnosis of LLT $[1,5]$. In our dogs, a hypoechoic, enlarged and hepatized lung lobe, pleural effusion and portions filled with scattered reverberating foci consistent with gas were observed by CUS. Additionally, presumptive dilated vascular structures appeared in the central portion of the lung lobe in 2 of 3 dogs. A color Doppler study was not useful due to respiratory motion artifact. Color Doppler ultrasonography could be limited to demonstrate flow in LLT, because of flow velocity, vessel size, the depth of lesion, scanner sensitivity and operator control. Moreover, any 


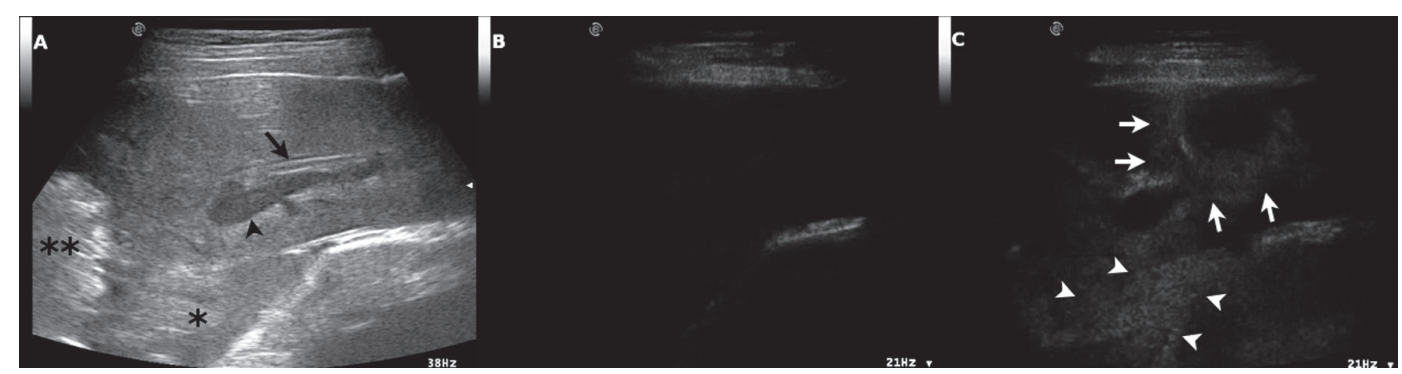

Fig. 1. Lung lobe torsion in case 1. B-mode ultrasound image (A) showing consolidated lung lobe. A fluid bronchogram (arrow) and dilated vascular structure (arrowhead) can be seen in the central portion of the consolidated lung lobe. A partial consolidation of another lung lobe can be seen to the left of the image $(*)$. Contrast harmonic mode ultrasound image of the same lesions before the injection of the contrast agent (B). Contrast-enhanced ultrasound images showing an inhomogeneous vascular enhancement (arrows) after 28 sec from injection of the contrast agent $(\mathrm{C})$. The other consolidated lung lobe shows a homogeneous and marked vascular enhancement (arrowheads) compared to the twisted lung lobe. $* *$ Reverberating foci consistent with gas.

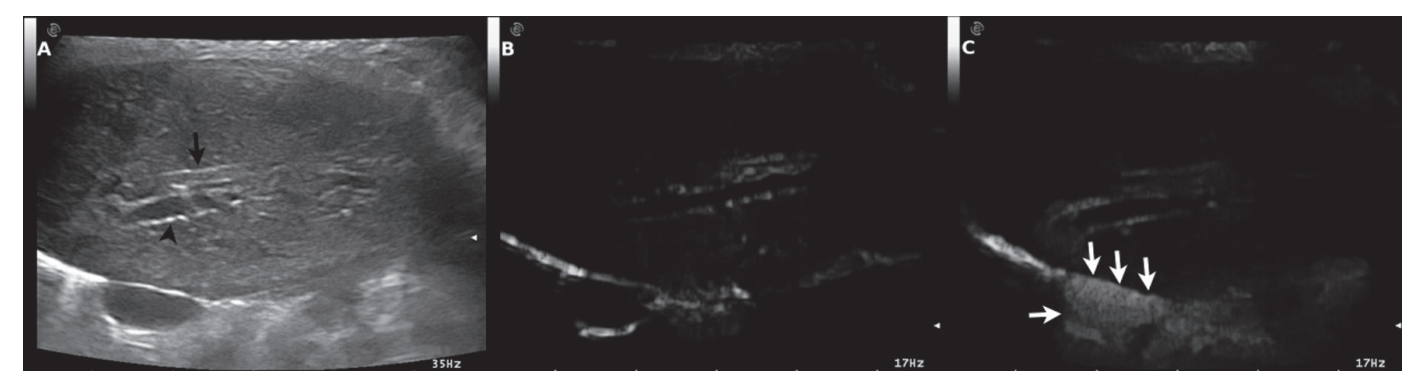

Fig. 2. Lung lobe torsion in case 2. B-mode ultrasound image (A) showing consolidated lung lobe. A fluid bronchogram (arrow) and dilated vascular structure (arrowhead) can be seen in the central portion of the consolidated lung lobe. Contrast harmonic mode ultrasound image of the same lesions before the injection of the contrast agent (B). Contrast-enhanced ultrasound images showing absence of vascular enhancement after injection of the contrast agent within twisted lung lobe and evident vascular enhancement (vena cava) at the periphery of lung lobe (arrows) after $28 \mathrm{sec}$ from injection of the contrast agent (C).

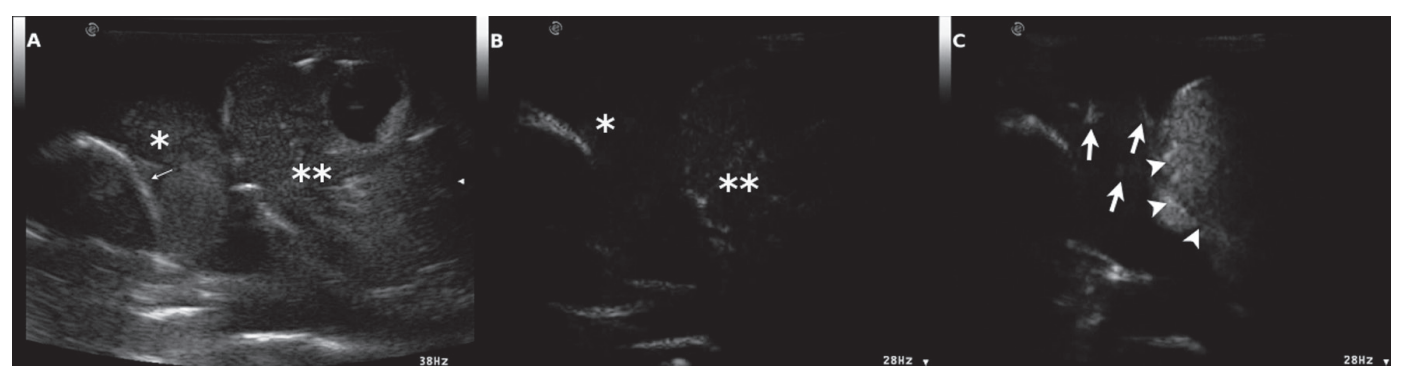

Fig. 3. Lung lobe torsion in case 3. B-mode ultrasound image (A) showing consolidated lung lobe $(*)$. Air bronchogram (thin arrow) within the suspected lung lobe is evident. Liver $(* *)$ can be seen to the right of the image. Contrast harmonic mode ultrasound image of the same scan before the injection of the contrast agent (B). Contrast-enhanced ultrasound images showing enhanced vessels inside the lung lobe (arrows) after $25 \mathrm{sec}$ from injection of the contrast agent (C). Vascular enhancement (arrowheads) can be seen within the liver (C).

motion of the tissue produces a Doppler shift. To detect the changes of blood flow in LLT (helpful finding in diagnostic process) and to overcome the limits of the Doppler modality, the authors used CEUS. Lack of contrast enhancement after contrast medium administration during a CT study can be seen when a lung lobe is completely rotated $[15,16]$. In case 2, CEUS showed the absence of vascular enhancement within the twisted lung lobe $\left(360^{\circ}\right.$ torsion). Incomplete LLT or twisted lung lobe at early stage can still show an arterial blood supply during a contrast-enhanced CT study [16]. In case 1, CEUS showed an inhomogeneous and mild enhancement of the partially twisted lung lobe $\left(180^{\circ}\right.$ torsion $)$ in agreement with previously reported contrast-enhanced CT findings [16]. In case 3, enhanced vessels were visualized during a CEUS study, although the twisted lung lobe was rotated approximately $360^{\circ}$. This finding was in agreement with persistent arterial blood supply within the twisted lung lobe, but clinical signs were present for 1 week and 
the twisted lung lobe appeared to be already necrotic during surgical exploration. Clinical and surgical features were not in accordance with a short-time duration of the torsion. In a previous study [15], twisted lung lobes with evidence of necrosis showed contrast enhancement after iodine intravenous injection during a CT study. The authors suggested that an arterial blood supply could be present also in the early stages of necrosis [15]. Further studies are needed to investigate the enhancement patterns in relation to the rotation degree of the twisted lung lobe and/or the time of torsion occurrence.

Lack of contrast enhancement can also be observed in other lung lesions, such as thromboembolic, cystic or necrotic conditions $[3,13]$. The lesions present in these cases are usually focal and limited to certain portions of the lung lobe. In cases of LLT, however, the entire lobe is affected [16].

In case 1, the CEUS revealed a homogeneous and marked enhancement of a second partially consolidated lung lobe: during surgery, the left caudal lobe showed an area of atelectasis which disappeared after the re-inflation using positive-pressure ventilation. These findings confirmed the usefulness of the CEUS in distinguishing pulmonary atelectasis from LLT.

CT is a useful diagnostic tool in cases of suspected LLT where the radiographic or ultrasonographic findings are non specific $[15,16]$, but it is not widely available, can be cost prohibitive, and requires general anesthesia and ionizing radiation. On CT, case 2 showed the absence of enhancement within the suspected twisted lung lobe after the contrast agent injection. Absence of vascular enhancement within the twisted lung lobe was also observed by CEUS. In case 2, contrast-enhanced CT also showed a peripheral ring-enhancement probably due to the inflammatory hypervascularization of the visceral pleura. This finding was not clearly observed by CEUS. Probably, the assessment of the blood perfusion in the visceral pleura was difficult, because of the hyperintensity of this tissue during a CEUS study. In case 3, the suspected twisted lung lobe showed mild contrastenhancement on a CT study in accordance with CEUS findings.

Considering our preliminary findings, CEUS can provide additional information to the CUS, in particular when a Doppler study is hampered by respiratory motion artifact. CEUS features of the present report, in accordance with previous CT and CUS studies $[1,5,15,16]$, suggest that the absence or reduction of the blood flow can be considered a specific finding in a suspected twisted lung lobe. Assessment of this change in pulmonary blood flow by CEUS, in association with radiographic and CUS findings, can increase the index of suspicion in LLT, reserving use of advanced diagnostic tools, as CT, only for "atypical" and limited cases. Further studies discerning the value of the CEUS in other lung diseases are necessary in order to compare them to the findings in this report.

In conclusion, the results of this case report indicate that qualitative analysis of the CEUS can improve the accuracy of CUS for detection of pulmonary blood flow compromise and should be considered in the diagnostic algorithm in dogs with LLT. Moreover, our report opens up new perspectives for assessing of pulmonary lesions using CEUS.
ACKNOWLEDGMENT. The authors wish to thank Jennifer A. Safko for her help during the preparation of the manuscript.

\section{REFERENCES}

1. Agut, A., Carrillo, J. D., Seva, J., Soler, M. and Laredo, F. G. 2013. What is your diagnosis? Lung lobe torsion. J. Am. Vet. Med. Assoc. 243: 333-335. [Medline] [CrossRef]

2. Bargellini, P., Orlandi, R., Paloni, C., Rubini, G., Fonti, P., Peterson, M. E. and Boiti, C. 2013. Contrast-enhanced ultrasonographic characteristics of adrenal glands in dogs with pituitarydependent hyperadrenocorticism. Vet. Radiol. Ultrasound 54: 283-292. [Medline] [CrossRef]

3. Caremani, M., Benci, A., Lapini, L., Tacconi, D., Caremani, A., Ciccotosto, C. and Magnolfi, A. L. 2008. Contrast enhanced ultrasonography (CEUS) in peripheral lung lesions: a study of 60 cases. J. Ultrasound 11: 89-96. [Medline] [CrossRef]

4. Choi, J. and Yoon, J. 2006. Lung lobe torsion in a Yorkshire terrier. J. Small Anim. Pract. 47: 557. [Medline] [CrossRef]

5. d'Anjou, M. A., Tidwell, A. S. and Hecht, S. 2005. Radiographic diagnosis of lung lobe torsion. Vet. Radiol. Ultrasound 46: 478-484. [Medline] [CrossRef]

6. Fan, Z., Li, Y., Yan, K., Wu, W., Yin, S., Yang, W., Xing, B., Li, X. and Zhang, X. 2013. Application of contrast-enhanced ultrasound in the diagnosis of solid pancreatic lesions - a comparison of conventional ultrasound and contrast-enhanced CT. Eur. J. Radiol. 82: 1385-1390. [Medline] [CrossRef]

7. Görg, C., Bert, T. and Kring, R. 2006. Contrast-enhanced sonography of the lung for differential diagnosis of atelectasis. $J$. Ultrasound Med. 25: 35-39. [Medline]

8. Haers, H. and Saunders, J. H. 2009. Review of clinical characteristics and applications of contrast-enhanced ultrasonography in dogs. J. Am. Vet. Med. Assoc. 234: 460-470. [Medline] [CrossRef]

9. Hofeling, A. D., Jackson, A. H., Alsup, J. C. and O'keefe, D. 2004. Spontaneous midlobar lung lobe torsion in a 2-year-old Newfoundland. J. Am. Anim. Hosp. Assoc. 40: 220-223. [Medline] [CrossRef]

10. Kurach, L. M., Nykamp, S. G. and Ringwood, P. B. 2012. What is your diagnosis? Lung lobe torsion. J. Am. Vet. Med. Assoc. 241: 1149-1151. [Medline] [CrossRef]

11. Murphy, K. A. and Brisson, B. A. 2006. Evaluation of lung lobe torsion in Pugs: 7 cases (1991-2004). J. Am. Vet. Med. Assoc. 228: 86-90. [Medline] [CrossRef]

12. Neath, P. J., Brockman, D. J. and King, L. G. 2000. Lung lobe torsion in dogs: 22 cases (1981-1999). J. Am. Vet. Med. Assoc. 217: 1041-1044. [Medline] [CrossRef]

13. Sartori, S., Postorivo, S., Vece, F. D., Ermili, F., Tassinari, D. and Tombesi, P. 2013. Contrast-enhanced ultrasonography in peripheral lung consolidations: what's its actual role? World J. Radiol. 5: 372-380. [Medline] [CrossRef]

14. Sartori, S. and Tombesi, P. 2010. Emerging roles for transthoracic ultrasonography in pulmonary diseases. World J. Radiol. 2: 203-214. [Medline] [CrossRef]

15. Schultz, R. M., Peters, J. and Zwingenberger, A. 2009. Radiography, computed tomography and virtual bronchoscopy in four dogs and two cats with lung lobe torsion. J. Small Anim. Pract. 50: 360-363. [Medline] [CrossRef]

16. Seiler, G., Schwarz, T., Vignoli, M. and Rodriguez, D. 2008. Computed tomographic features of lung lobe torsion. Vet. Radiol. Ultrasound 49: 504-508. [Medline] [CrossRef]

17. Thorelius, L. 2004. Contrast-enhanced ultrasound for extrahepatic lesions: preliminary experience. Eur. J. Radiol. 51: S31-38. [Medline] [CrossRef] 